\title{
Visitas domiciliares no Brasil: características da atividade basilar dos Agentes Comunitários de Saúde
}

\author{
Home Visits in Brazil: characteristics of the baseline activity of \\ Community Health Workers \\ Cristiane Abdon Nunes', Rosana Aquino², Maria Guadalupe Medinaㄹ, Ana Luiza Queiroz \\ Vilasbôas ${ }^{\mathbf{4}}$, Elzo Pereira Pinto Júnior $\mathbf{5}$, Leandro Alves da Luz $\mathbf{6}$
}

1 Universidade Federal da Bahia (UFBA), Instituto de Saúde Coletiva (ISC) Salvador (BA), Brasil. Orcid: https://orcid. org/0000-0001-63529858

abdoncris@gmail.com

2 Universidade Federal da Bahia (UFBA), Instituto de Saúde Coletiva (ISC) Salvador (BA), Brasil. Orcid: http://orcid. org/0000-0003-39065170

aquino@ufba.br

3 Universidade Federal da Bahia (UFBA), Instituto de Saúde Coletiva (ISC) Salvador (BA), Brasil. Orcid: https://orcid. org/0000-0001-72832947

medina@ufba.br

${ }^{4}$ Universidade Federal da Bahia (UFBA), Instituto de Saúde Coletiva (ISC) Salvador (Ba), Brasil. Orcid: https://orcid. org/0000-0002-55668337

analuvilas@gmail.com

5 Universidade Federal da Bahia (UFBA), Instituto de Saúde Coletiva (ISC) Salvador (BA), Brasil. Orcid: https://orcid. org/0000-0002-69772071

elzojr@hotmail.com

${ }^{6}$ Universidade Federal da Bahia (UFBA), Instituto de Saúde Coletiva (ISC) -

Salvador (Ba), Brasil.

Orcid: https://orcid.

org/0000-0002-3566-

3117

leojluz@gmail.com
DOI: 10.1590/0103-11042018S209

RESUMO Caracterização das visitas domiciliares realizadas pelos Agentes Comunitários de Saúde no Brasil. Estudo transversal, amostragem aleatória por conglomerados. 1.526 agentes entrevistados em 100 municípios, com representatividade nacional. A maioria dos agentes era composta por mulheres, com ensino médio completo e mediana de idade de 39 anos. Visitas domiciliares são realizadas frequentemente por $99,1 \%$ dos agentes, sendo que $67,4 \%$ fazem pelo menos uma visita mensal. Visitar famílias com menores de um ano de idade e gestantes de risco até 15 dias foi referido por 70,4\% dos agentes; 40,3\% afirmaram programar frequentemente visitas com profissionais da unidade; $80,9 \%$ adotavam critérios para sua realização. Nas visitas, mais de $45 \%$ orientavam sobre serviços de saúde e verificavam cartão de vacinação; quase um terço questionava sobre doenças prevalentes; entre $20 \%$ e $25 \%$ entregavam e orientavam o uso de medicamentos, pesavam crianças e cadastravam famílias; menos de $20 \%$ verificavam condições ambientais e realizavam busca ativa de faltosos. Essas falhas podem estar associadas à baixa participação da equipe no planejamento da visita, indicando problemas na organização do processo de trabalho dos agentes. Destaca-se a importância da Visita Domiciliar no desenvolvimento de ações de promoção da saúde e prevenção de agravos no âmbito da Atenção Primária à Saúde.

PALAVRAS-CHAVE Agentes Comunitários de Saúde. Atenção Primária à Saúde. Visita domiciliar.

ABSTRACT Characterization of home visits performed by Community Health Workers in Brazil. Cross-sectional study, with random sampling by clusters. 1.526 agents interviewed, distributed in 100 municipalities. The majority of the agents were women, with complete secondary education and a median age of 39 years. Households visits are frequently performed by $99.1 \%$ of the agents, with $67.4 \%$ making at least one monthly visit. Visiting families with infants and pregnant women at intervals of up to 15 days was reported by $70.4 \%$ of the agents; while frequently scheduled visits with other professionals from the unit were reported by $40.3 \%$; and $80.9 \%$ adopted criteria for its accomplishment. During visits, more than $45 \%$ of the agents provided information about health services and reviewed vaccination cards; between $20 \%$ and $25 \%$ delivered and guided the use of medicines, weighed children and registered families; less than $20 \%$ verified environmental 
conditions and actively searched for defaulters. These shortfalls may be associated with low team participation during the visit's planning, indicating problems in the organization of the agents' work process. Nonetheless, the importance of home visits in the development of actions of health promotion and injury prevention within the scope of Primary Health Care is highlighted.

KEYWORDS Community Health Workers. Primary Healthy Care. Home calls.

\section{Introdução}

No Brasil, o século XX foi marcado por diversas experiências de criação e expansão de serviços de atenção voltados para os cuidados primários em saúde. Contudo, foi no início da década de 1990 que surgiram as bases do que, posteriormente, viria a se constituir em uma política de saúde de abrangência nacional, tendo a Atenção Primária à Saúde (APS) como eixo norteador de todo o sistema de saúde com a implantação do Programa de Agentes Comunitários de Saúde (Pacs) e do Programa Saúde da Família (PSF), que passou, a partir de 1997, a ser denominado Estratégia Saúde da Família (ESF), modelo prioritário de reorganização da APS no País, segundo as normativas federais do Sistema Único de Saúde (SUS) $\mathbf{1}^{\mathbf{2}}$.

Diversos estudos de abrangência nacional evidenciaram o impacto da saúde da família no estado de saúde da população, a exemplo da redução da mortalidade infantil e de menores de $5 \operatorname{anos}^{3-5}$ e da redução das hospitalizações por causas sensíveis à atenção primária ${ }^{6}$, indicando o acerto dessa política na consolidação da APS enquanto modelo estruturante do sistema de saúde.

Alguns autores demonstraram que, até 1997, a expansão numérica da APS se deveu, principalmente, à expansão do Pacs, implantado em mais de 2.200 municípios. A partir de 1998, o PSF alcançou abrangência nacional, sendo implantado em mais de mil municípios do País. Desde então, observou-se ampliação quantitativa do número de Agentes Comunitários de Saúde (ACS) e de Equipes de Saúde da Família (EqSF), caracterizando uma crescente expansão de cobertura em grande parte dos municípios e ampliação para estados e regiões onde a estratégia não existia anteriormente, alcançando quase a totalidade dos municípios brasileiros ${ }^{\mathbf{1}}$. Hoje, o Brasil possui o maior número de ACS no mundo, os chamados Community Health Workers, ultrapassando a marca de $260.000^{7}$.

O Pacs foi criado pelo Ministério da Saúde em 1991 para intervir sobre as altas taxas de mortalidade infantil e materna na Região Nordeste, e logo foi ampliado para a Região Norte, em situação de emergência sanitária devido à epidemia de cólera. Desde a criação do Programa, os agentes comunitários têm sido identificados como mediadores entre a comunidade e os serviços de saúde, atuando por meio de ações educativas, prevenção de agravos e de promoção da saúde. As atribuições dos ACS, definidas pela Portaria $\mathrm{n}^{\circ}$ 1.886 , de $1997^{8}$, foram reiteradas na edição da Política Nacional de Atenção Básica (PNAB), de $2011^{9}$. Em paralelo, o processo de regulamentação da profissão aconteceu em 2002, com a promulgação da Lei $\mathrm{n}^{0} 10.507^{10}$, e vem passando por reformulações que, às vezes, contrapõem-se a atribuições da polítican". Entre as atribuições dos ACS presentes na PNAB 2011, destacam-se: realizar o cadastramento e a orientação das famílias quanto 
aos serviços de saúde disponíveis; acompanhar, por meio de Visita Domiciliar (VD), todas as famílias e indivíduos sob sua responsabilidade; desenvolver ações educativas visando à promoção da saúde, à prevenção das doenças e ao acompanhamento das pessoas com problemas de saúde, além de promover a integração entre a equipe de saúde e a população adstrita 9 .

No cenário de reorganização dos serviços de saúde, a partir da APS, seja no âmbito das EqSF ou nas Unidades Básicas de Saúde, diversos estudos apontam para os agentes comunitários como elementos nucleares para o desenvolvimento das ações de saúde ${ }^{12-14}$, problematizando suas atribuições ${ }^{\mathbf{1 4}, 15}$, práticas/processo de trabalho ${ }^{\mathbf{1 3}, 16-18}$, condições de trabalho $\mathbf{1 2}^{\mathbf{1 9}, 20}$, entre outros aspectos.

Mais do que meros suportes para execução de ações educativas em saúde, os ACS têm sido considerados participantes efetivos do trabalho no SUS. A literatura sobre a contribuição desse profissional para a consolidação do SUS problematizou a existência de duas dimensões principais da atuação do ACS: uma dimensão técnica, relativa ao atendimento dos indivíduos e das famílias, a intervenção para prevenção de agravos ou para o monitoramento de grupos ou problemas específicos; e uma dimensão política, no sentido de organização da comunidade e de transformação das condições de vida que levam aos problemas de saúde.

Outro aspecto mencionado diz respeito às dificuldades para cumprir suas atribuições, em função do amplo leque de exigências e das limitadas condições socioeconômicas das famílias acompanhadas ${ }^{21}$. Contudo, é justamente no contexto dos limites enfrentados no cotidiano do seu trabalho que o ACS se inscreve de forma privilegiada na dinâmica de implantação e de consolidação do novo modelo de saúde, dado que, atuando numa posição estratégica de mediador entre a comunidade e o serviço de saúde, ora ele pode funcionar como facilitador, ora como empecilho nesse processo ${ }^{22}$.

Entre as atribuições do ACS, destaca-se a realização da VD, que tem como objetivo viabilizar o acompanhamento das famílias e dos indivíduos de forma integral ${ }^{9}$. Diferentes autores atribuem à VD a concepção de instrumento principal do trabalho do ACS, sendo uma das suas atividades mais importantes ${ }^{\mathbf{2 3 , 2 4}}$, fato também referido pelos agentes comunitários 25-27. Corroborando isso, estudos apontaram que a VD foi importante para a construção de novas relações entre profissionais e familiares, formação de vínculos, aumento do acesso a serviços de saúde, maior acesso às informações sobre os serviços disponíveis e melhor compreensão das necessidades e dos contextos das famílias acompanhadas 28-30.

Ao longo dos 30 anos de constituição e implementação do SUS, e num contexto de luta pela consolidação da ESF enquanto política pública prioritária para a reorganização da atenção primária no País, faz-se necessário discutir suas práticas. Neste artigo, em particular, tratou-se de descrever o perfil socioeconômico e demográfico dos ACS do Brasil e analisar as práticas de VD segundo região do País e localização das unidades de saúde, zona urbana e rural, à luz da PNAB, publicada em 2011 e vigente até setembro de 2017.

\section{Material e métodos}

$\mathrm{O}$ desenho de estudo foi um inquérito nacional, realizado em 2015, cuja unidade de análise foram os ACS. A população de estudo contemplou todos os ACS distribuídos nas unidades de saúde de APS no Brasil, que totalizavam 255.772, com base no censo de unidades básicas de saúde realizado em 2012 ${ }^{31}$. Foi realizada uma amostragem aleatória por conglomerados com representatividade nacional, obtida através de sorteio por estágios sucessivos, inicialmente, de 100 municípios participantes, de acordo com a distribuição proporcional das unidades de saúde nas cinco regiões brasileiras; seguido de sorteio das unidades de saúde e das equipes de 
saúde. Todos os ACS das EqSF selecionadas foram incluídos, exceto os que estavam em desvio de função, férias, afastamento por doença e licença maternidade ou ausentes nas unidades por motivo de agenda; e, no caso das unidades de atenção básica convencional, oito ACS foram selecionados por meio de sorteio simples. A amostra final foi composta por 1.526 ACS.

Foi aplicado um questionário estruturado aos ACS nas unidades de saúde selecionadas, elaborado a partir de revisão de literatura e da análise documental de instrumentos normativos federais vigentes à época da coleta de dados 9 , sobre o trabalho do agente comunitário. Os entrevistadores foram devidamente treinados e supervisionados por assistentes de pesquisa em campo.

As variáveis do perfil socioeconômico e demográfico dos ACS foram: sexo; idade; escolaridade (fundamental incompleto/completo, ensino médio completo e superior incompleto/ completo); raça/cor; renda familiar em salários mínimos (até 1, de 1 a 1,5 e mais de 1,5); e número de famílias cadastradas na microárea.

As variáveis relacionadas às visitas domiciliares foram: realiza VD (sempre/na maioria das vezes, algumas vezes/raras vezes/nunca); número de turnos de trabalho dedicados a VD (menos de cinco, cinco ou mais); frequência das visitas domiciliares de rotina às famílias (uma visita pelo menos uma vez por mês, uma visita em intervalo maior que um mês e sem periodicidade definida a depender da condição do usuário); frequência das visitas domiciliares às famílias com crianças menores de 1 ano ou gestante de risco (uma visita a cada 15 dias, uma visita por mês, uma visita em intervalo maior que um mês); visitas domiciliares programadas com os demais profissionais da unidade (sim, sempre/na maioria das vezes, algumas/ raras vezes/nunca); categoria do profissional (médico, enfermeiro, cirurgião-dentista); existência de critérios definidos para realização das visitas (sim, não); critérios utilizados (residir em área distante, residir em área de difícil acesso, famílias com presença de idosos, pessoas com dificuldade de locomoção, portadores de doença crônica, crianças $<2$ anos e gestantes); e atividades realizadas nas visitas (orienta sobre os serviços de saúde, cadastra as famílias, verifica cartão de vacinação, levanta/acompanha casos de doenças, acompanha as famílias no Programa Bolsa Família (PBF), busca ativa de faltosos, verifica condições ambientais, entrega medicamentos e orienta quanto ao uso, pesa as crianças).

No caso do tipo de critério e das atividades realizadas, inicialmente, eram assinaladas as alternativas referidas espontaneamente pelos entrevistados e, a seguir, era questionada cada uma das alternativas não referidas. A presente análise só considerou as respostas espontâneas dos ACS.

Foi realizada análise descritiva, segundo a localização das unidades de saúde por região e zona urbana e rural, com apresentação tabular e gráfica, utilizando o programa Stata versão 12.0 e o software Excel 2013. O projeto foi submetido e aprovado pelo Comitê de Ética em Pesquisa do Instituto de Saúde Coletiva da Universidade Federal da Bahia (Parecer no 871.501/2014).

\section{Resultados e discussão}

\section{Perfil dos ACS - aspectos importan- tes sobre a especificidade e a abran- gência de seu trabalho}

No Brasil, em 2015, mais de $80 \%$ dos ACS eram do sexo feminino ( $83,2 \%$ ), percentual que variou entre as regiões, de $76,4 \%$ (Nordeste) a 91,8\% (Sul), e foi menor na área rural $(72,5 \%)$ do que na área urbana $(86,1 \%)$. A mediana de idade foi de 39 anos, com pouca variação entre os estratos estudados. Quase 65\% se autodeclararam de cor parda $(52,9 \%)$ ou preta $(12,0)$, com grande variação entre as regiões, sendo quase $85 \%$ de pardos 
e pretos na Região Norte e cerca de $30 \%$ na Região Sul. Mais de $80 \%$ referiram possuir ensino médio completo, e $18,8 \%$, superior incompleto ou completo, sendo o último percentual menor na Região Sul (10,5\%) e na zona rural $(8,4 \%)$, onde foram maiores os percentuais de ensino médio completo. Mais de $70 \%$ referiram renda familiar entre 1 e 1,5 salário mínimo, e quase $16 \%$, de até 1 salário mínimo, sendo observados menores níveis de renda nas Regiões Centro-Oeste, Sudeste e áreas rurais, onde os percentuais de agentes com até 1 salário mínimo foram superiores a $20 \%$ (tabela 1 ).

Mais de $80 \%$ residiam em sua área de atuação, variando entre $76,4 \%$ na Região Norte e $88,6 \%$ no Sul, $80,5 \%$ na zona urbana e $88,8 \%$ na zona rural. A mediana do número de famílias cadastradas na microárea de atuação foi de 150, bem menor na área rural do que na urbana (95 e 160, respectivamente), variando de 139 (Centro-Oeste) a 192 (Sul), entre as regiões (tabela 1).

Tabela 1. Perfil socioeconômico dos Agentes Comunitários de Saúde, segundo localização da unidade de saúde, Regiões e Brasil, 2015

\begin{tabular}{|c|c|c|c|c|c|c|c|c|}
\hline \multirow{3}{*}{ Variáveis } & \multirow[b]{2}{*}{ Brasil } & \multicolumn{5}{|c|}{ Região } & \multicolumn{2}{|c|}{ Localização } \\
\hline & & Norte & Nordeste & Centro-Oeste & Sudeste & Sul & Rural & Urbana \\
\hline & $\%$ & $\%$ & $\%$ & $\%$ & $\%$ & $\%$ & $\%$ & $\%$ \\
\hline \multicolumn{9}{|l|}{ Sexo } \\
\hline Feminino & 83,2 & 84,9 & 76,4 & 85,6 & 85,7 & 91,8 & 72,5 & 86,1 \\
\hline Masculino & 16,8 & 15,1 & 23,6 & 14,4 & 14,20 & 8,2 & 27,5 & 13,9 \\
\hline Idade (mediana) & 39,0 & 39,0 & 40,0 & 38,0 & 37,0 & 39,0 & 38,0 & 39,0 \\
\hline \multicolumn{9}{|l|}{ Escolaridade (Maior nível) } \\
\hline Fundamental incompleto/completo & 1,1 & 0,0 & 1,7 & 1,6 & 0,9 & 0,5 & 1,3 & 1,0 \\
\hline Ensino médio completo & 80,1 & 76,4 & 78,3 & 77,6 & 80,4 & 89,0 & 90,3 & 77,5 \\
\hline Superior em curso/completo & 18,8 & 23,6 & 20,0 & 20,8 & 18,8 & 10,5 & 8,4 & 21,6 \\
\hline \multicolumn{9}{|l|}{ Raça/Cor } \\
\hline Branca & 33,3 & 12,1 & 20,4 & 24,0 & 44,5 & 67,0 & 32,9 & 33,4 \\
\hline Preta & 12,0 & 15,7 & 11,8 & 16,8 & 11,5 & 7,3 & 6,9 & 13,0 \\
\hline Parda & 52,9 & 68,7 & 66,2 & 56,8 & 43,2 & 23,9 & 56,6 & 51,2 \\
\hline Outra & 1,8 & 3,5 & 1,7 & 2,4 & 0,9 & 1,8 & 0,6 & 2,1 \\
\hline \multicolumn{9}{|l|}{ Renda familiar } \\
\hline Até 1 salário mínimo & 15,8 & 13,3 & 17,07 & 21,6 & 20,1 & 3,2 & 21,7 & 14,3 \\
\hline 1 - 1,5 salário mínimo & 73,8 & 75,5 & 70,0 & 59,2 & 75,0 & 87,2 & 69,8 & 74,8 \\
\hline > 1,5 salário mínimo & 10,4 & 11,2 & 12,95 & 19,2 & 4,9 & 9,6 & 8,5 & 10,9 \\
\hline Mora na área de atuação & 82,2 & 76,4 & 84,5 & 79,2 & 79,9 & 88,6 & 88,8 & 80,5 \\
\hline Famílias cadastradas (mediana) & 150,0 & 143,0 & 140,0 & 139,0 & 153,0 & 192,0 & 95,0 & 160,0 \\
\hline
\end{tabular}




\section{Frequência, periodicidade e planeja- mento das visitas domiciliares}

A VD é uma atividade central do processo de trabalho do ACS e deve ser realizada com frequência média de uma visita família/mês, sendo que as famílias com maior necessidade deverão ser visitadas com mais frequência9. Nesta pesquisa, foram consideradas a realização e a frequência da VD a famílias e a grupos estratégicos (crianças e gestantes) e turnos dedicados a essa atividade. Os resultados apontaram que, aproximadamente, a totalidade dos ACS realiza visitas 'sempre' e 'na maioria das vezes' (99,1\%) às famílias ou pessoas de sua microárea, não havendo diferenças entre as regiões e entre as zonas urbana e rural. No que se refere aos turnos de trabalho, 74,4\% dos agentes comunitários declararam destinar cinco turnos ou mais a essa atividade, com maiores proporções na região Sul $(93,6 \%)$ e menores no CentroOeste $(62,4 \%)$, e menores proporções na zona urbana $(73,3 \%)$ do que na zona rural (78,4\%) (tabela 2).

Estudos sobre a frequência de VD no estado de São Paulo e Minas Gerais apontaram que, respectivamente, $85,5 \%$ e $99,6 \%$ dos ACS realizavam a atividade regularmente, com percentuais bastante próximos ao encontrado nesta pesquisa ${ }^{14,32}$. Fracolli33 e Lara $^{34}$ também encontraram resultados semelhantes para a regularidade na realização de VD pelos ACS, e, assim como no presente inquérito, a maioria dos profissionais utilizava cinco ou mais turnos de trabalho para esse fim.

Com relação à periodicidade das visitas de rotina às famílias, 67,4\% dos ACS afirmaram pelo menos uma visita mensal, com percentuais semelhantes entre zona urbana e rural, sendo observada a maior proporção na Região Centro-Oeste (83,2\%) e a menor na Região Sudeste (57,4\%). Ressalta-se, ainda, que, apesar da maioria dos ACS cumprir a recomendação de média mensal de pelo menos uma VD por família, quase um terço deles relatou não seguir uma periodicidade definida para a realização de visitas, vinculando essa atividade à condição de saúde dos usuários, sem diferenças entre áreas urbanas e rurais, com maiores percentuais na Região Sudeste (39,7\%) e menores nas Regiões Centro-Oeste (15,2\%), Sul (25,1\%) e Nordeste (25,4\%) (tabela 2).

Tabela 2. Percentual de agentes comunitários que realiza visita, que dedica cinco ou mais turnos de trabalho a essa atividade e periodicidade das visitas às famílias segundo localização da unidade de saúde e Brasil, 2015

\begin{tabular}{|c|c|c|c|c|c|c|}
\hline \multirow{3}{*}{$\begin{array}{l}\text { Localização da } \\
\text { unidade de saúde }\end{array}$} & \multirow[b]{2}{*}{ Total } & \multirow[b]{2}{*}{$\begin{array}{r}\text { Sempre } \\
\text { realiza visita } \\
\text { domiciliar }\end{array}$} & \multirow[b]{2}{*}{$\begin{array}{r}\text { Dedica } 5 \\
\text { turnos ou mais } \\
\text { para atividade }\end{array}$} & \multicolumn{3}{|c|}{ Periodicidade das visitas domiciliares } \\
\hline & & & & $\begin{array}{r}\text { Pelo menos } \\
\text { mensal para } \\
\text { visitas de } \\
\text { rotina }\end{array}$ & $\begin{array}{r}\text { Depende da } \\
\text { condição do } \\
\text { usuário }\end{array}$ & $\begin{array}{r}\text { Até } 15 \text { dias para } \\
\text { famílias com } \\
\text { crianças < } 1 \text { ano e } \\
\text { gestantes de risco }\end{array}$ \\
\hline & $\mathbf{N}$ & $\%$ & $\%$ & $\%$ & $\%$ & $\%$ \\
\hline Brasil & 1.526 & 99,1 & 74,4 & 67,4 & 29,5 & 70,4 \\
\hline Região Norte & 199 & 99,5 & 65,3 & 62,8 & 31,2 & 72,9 \\
\hline Região Nordeste & 535 & 99,1 & 68,6 & 72,7 & 25,4 & 82,2 \\
\hline Região Centro-Oeste & 125 & 100,0 & 62,4 & 83,2 & 15,2 & 70,4 \\
\hline Região Sudeste & 448 & 98,9 & 79,2 & 57,4 & 39,7 & 63,6 \\
\hline Região Sul & 219 & 98,6 & 93,6 & 69,9 & 25,1 & 53,4 \\
\hline Zona Urbana & 1.206 & 99,0 & 73,3 & 67,1 & 29,3 & 67,2 \\
\hline Zona Rural & 320 & 99,4 & 78,4 & 68,4 & 30,3 & 82,8 \\
\hline
\end{tabular}


Condicionar a visita apenas ao quadro de saúde do usuário revela inconsistências na definição de critérios e na concepção da VD, que deve ser um instrumento para promover o cuidado em saúde de forma ampliada, para além do monitoramento de doenças e agravos. De um lado, a falta de critérios pode corroborar a fragmentação dessa atividade e a ocorrência de visitas em intervalos muito superiores ao recomendado, inclusive no caso de famílias que necessitam de um acompanhamento sistemático e intensivo. Do outro, o modelo de atenção centrado na doença condiciona a lógica das práticas à demanda espontânea e reduz o potencial do ACS na identificação, na intervenção e no encaminhamento oportuno de situações que exigem cuidados preventivos e diagnóstico precoce e na implementação de ações de promoção da saúde.

A variação na periodicidade das visitas tem sido observada em diversos estudos. Oliveira $^{35}$ evidenciou que $25 \%$ das famílias investigadas em seu estudo recebiam visitas em intervalos maiores de dois meses ou nunca foram visitadas. Para o autor, uma das explicações para esse fato estaria relacionada à acumulação de outras atividades concomitantes, como cadastramento do cartão SUS, do Programa de Segurança Alimentar e do Programa Bolsa Escola.

A sobrecarga de trabalho foi relatada pelos agentes como dificuldade para realização das visitas $^{36,37}$, sinalizando a falta de clareza das equipes quanto às principais atribuições dos ACS, deslocando-os para outras atividades que demandam tempo e dificultam o cumprimento das visitas domiciliares conforme o preconizado, ou até em maior frequência, em caso de necessidade.

No estudo de Gomes ${ }^{21}$, apenas $45,4 \%$ dos ACS visitavam todas as famílias no mês, ao passo que $27,3 \%$ não conseguiam cumprir essa recomendação em função de algum imprevisto ${ }^{21}$. Essa situação também tem refletido diretamente sobre a percepção das famílias e de outros profissionais, haja vista que usuários e enfermeiros mostraram-se insatisfeitos com o baixo número de famílias visitadas pelos $\mathrm{ACS}^{\mathbf{2 4}, \mathbf{3 8}}$.

Um estudo de metassíntese realizado sobre o trabalho dos ACS na ESF 12 identificou artigos em que a temática da 'falta de dimensionamento do trabalho dos ACS' foi abordada, destacando-se o excesso de funções previstas e a pouca clareza das suas atribuições. Costa ${ }^{14}$ evidenciou que $33,6 \%$ dos agentes comunitários referiram auxiliar o profissional de nível superior no ambiente clínico, assumindo tarefas como desinfecção e esterilização de instrumental clínico, que caberiam aos técnicos ou auxiliares de enfermagem, ou funções administrativas, como o agendamento de consultas e exames, a conferência de estoque de material de escritório e médico-hospitalar. Situações como essas, além de desviar o foco das ações no território, descaracterizam o papel do ACS na equipe de saúde e dificultam a consolidação de uma identidade profissional própria ${ }^{\mathbf{1 4}}$.

Com relação à periodicidade de VD às famílias com crianças menores de um ano de idade e gestante de risco, $70,4 \%$ dos agentes do presente inquérito afirmaram realizar tal atividade a cada 15 dias. Esses percentuais foram mais elevados na Região Nordeste $(82,2 \%)$ e menores na Região Sul (53,4\%), com maior percentual na zona rural $(82,8 \%)$ do que na zona urbana $(67,2 \%)$ (tabela 2). As visitas domiciliares devem ser realizadas com base em critérios de risco e vulnerabilidade, de modo que famílias com maior necessidade sejam visitadas mais vezes ${ }^{9}$. Tendo em vista que se trata de pessoas com necessidade de monitoramento mais intensivo, torna-se importante que a frequência de visitas a esse público supere a média recomendada na PNAB.

A programação conjunta da VD deve nortear a realização dessa atividade, sendo, portanto, uma etapa fundamental na definição da agenda de trabalho do ACS e da equipe multiprofissional de APS 9 . Quando questionados se a realização das visitas domiciliares era programada com os demais profissionais da unidade de saúde, 40,3\% dos ACS afirmaram programá-las 'sempre 
ou na maioria das vezes', e $38,2 \%$, 'algumas vezes ou raramente', sem grandes variações entre zona urbana e rural. Foram observadas diferenças entre os padrões de resposta de acordo com as regiões, ressaltando que apenas $28,3 \%$ dos agentes da Região Sul referiram sempre ou na maioria das vezes programar visitas com outros profissionais, enquanto quase $50 \%$ dos agentes da Região Norte referiram fazê-lo (tabela 3).

O planejamento conjunto das visitas possibilita, entre outros aspectos, qualificar o ACS para a abordagem da família, dado que permite obter, com antecedência, informações sobre a história do indivíduo ou da família a ser visitada e definir algumas das ações a serem realizadas. A baixa proporção de programação conjunta evidenciada neste estudo pode indicar a fragmentação do trabalho em equipe no planejamento da principal atividade que compõe o processo de trabalho do ACS.

Com relação à categoria profissional, 75,9\% afirmaram programar as visitas domiciliares com enfermeiros, $65,7 \%$ com médicos e $20,4 \%$ com dentistas. Entre as regiões, observou-se, na Região Sul, menores proporções de agentes que referiram programar visitas com enfermeiros
(59,4\%), médicos $(50,2 \%)$ e cirurgiões-dentistas (16,0\%); percentuais semelhantes foram observados entre as demais quatro regiões para programação de visitas com enfermeiros (entre $78,3 \%$ e $79,2 \%$ ), e maiores percentuais com médicos (75,4\%) e cirurgiões-dentistas (29,5\%) na Região Sudeste. No tocante à comparação entre zona urbana e zona rural, foram observadas diferenças na programação das visitas domiciliares com enfermeiros, médicos e cirurgiões-dentistas, sendo maiores as proporções para esses profissionais na zona urbana ( $76,6 \%, 67,2 \%$ e $21,8 \%$, respectivamente) do que na zona rural $(73,1 \%, 59,7 \%$ e $15,0 \%$, respectivamente) (tabela 3).

Outros estudos assinalaram a escassez de visitas realizadas conjuntamente, inclusive com profissionais médicos, enfermeiros e técnicos de enfermagem. Há menção a uma maior participação do profissional de assistência social ${ }^{39}$. Concordando com esses achados, Gaíva ${ }^{40}$ identificou que a prática da VD tem sido realizada de forma fragmentada, sem a presença de toda a equipe e focada no indivíduo doente ${ }^{40}$. A pouca interação entre enfermeiros e ACS e o planejamento assistemático têm sido relatados como pontos críticos dessa atividade ${ }^{37}$.

Tabela 3. Percentual de agentes comunitários que referiu programar as visitas domiciliares com outro profissional da equipe, por periodicidade e categoria profissional, segundo localização da unidade de saúde e Brasil, 2015

\begin{tabular}{|c|c|c|c|c|c|c|c|}
\hline \multirow{3}{*}{$\begin{array}{l}\text { Localização da } \\
\text { unidade de saúde }\end{array}$} & \multirow[b]{2}{*}{ Total } & \multicolumn{3}{|c|}{$\begin{array}{l}\text { Visita domiciliar programada com } \\
\text { outro profissional da equipe }\end{array}$} & \multicolumn{3}{|c|}{$\begin{array}{l}\text { Profissional de saúde que realiza } \\
\text { visita programada com agente }\end{array}$} \\
\hline & & $\begin{array}{r}\text { Sim, sempre ou } \\
\text { na maioria das } \\
\text { vezes }\end{array}$ & $\begin{array}{r}\text { Sim, algumas ou } \\
\text { raras vezes }\end{array}$ & $\begin{array}{c}\text { Nunca, não } \\
\text { sabe ou não } \\
\text { respondeu }\end{array}$ & Enfermeiro & Médico & $\begin{array}{r}\text { Cirurgião- } \\
\text { dentista }\end{array}$ \\
\hline & $\mathbf{N}$ & $\%$ & $\%$ & $\%$ & $\%$ & $\%$ & $\%$ \\
\hline Brasil & 1.526 & 40,3 & 38,2 & 21,5 & 75,9 & 65,7 & 20,4 \\
\hline Região Norte & 199 & 49,2 & 34,7 & 16,1 & 78,4 & 72,9 & 20,1 \\
\hline Região Nordeste & 535 & 42,1 & 37,0 & 20,9 & 78,3 & 59,8 & 16,6 \\
\hline Região Centro-Oeste & 125 & 34,4 & 48,0 & 17,6 & 78,4 & 71,2 & 24,8 \\
\hline Região Sudeste & 448 & 41,7 & 40,6 & 17,7 & 79,2 & 75,4 & 29,5 \\
\hline Região Sul & 219 & 28,3 & 33,8 & 37,9 & 59,4 & 50,2 & 16,0 \\
\hline Zona Urbana & 1.206 & 40,2 & 39,1 & 20,7 & 76,6 & 67,2 & 21,8 \\
\hline Zona Rural & 320 & 40,6 & 34,7 & 24,7 & 73,1 & 59,7 & 15,0 \\
\hline
\end{tabular}




\section{Critérios e atividades realizadas na VD}

A PNAB, vigente até 2017, embora estabeleça a frequência média mensal de visitas de ACS a serem realizadas em cada domicílio, não define os critérios a serem utilizados para orientar a sua periodicidade. Dado esse fato, cabe assinalar possíveis dificuldades na padronização e na priorização das visitas domiciliares, sejam elas realizadas exclusivamente pelos ACS ou conjuntamente com demais profissionais da equipe. A existência de critérios para realização de visitas domiciliares definidos pela equipe foi referida por $80,9 \%$ dos ACS entrevistados, variando entre as regiões de 70,4\%, no Centro-Oeste, a aproximadamente $82 \%$, no Norte, no Nordeste e no Sul, com pequenas diferenças entre zona urbana (80,5\%) e zona rural (82,2\%) (figura 1).

A respeito dos critérios referidos, mais de $30 \%$ dos agentes mencionaram a presença de: pessoas com dificuldade de locomoção $(53,7 \%)$, gestantes $(38,7 \%)$, portadores de doenças crônicas $(35,9 \%)$, idosos $(31,1 \%)$ e crianças menores de 2 anos (30,7\%), com percentuais superiores na zona rural, quando comparada com a zona urbana, exceto para o primeiro critério. Na comparação entre as regiões, o percentual de agentes que referiram como critério para VD a presença de pessoas com dificuldade de locomoção nas famílias foi superior a $50 \%$, exceto na Região Centro-Oeste (39,2\%). A presença de gestantes como critério variou, aproximadamente, de $34 \%$ (Sul e Centro-Oeste) a $44,5 \%$ (Nordeste), enquanto a de menores de 2 anos variou de $28 \%$ (Centro-Oeste) a $41,7 \%$ (Norte). A existência de portadores de doenças crônicas na família foi um critério para a visita do ACS, que variou de $26,4 \%$ (Centro-Oeste) a $38,7 \%$ (Norte), enquanto a de idosos oscilou de $18,7 \%$ (Sul) a $41,7 \%$ (Norte) (figura 1).

Menos de 5\% dos agentes referiram, espontaneamente, como critérios para realização de visitas domiciliares a família residir em área distante $(4,1 \%)$ ou de difícil acesso $(4,7 \%)$. Os percentuais para os dois critérios foram menores na Região Sul (1,8\% e $1,4 \%$, respectivamente) e maiores na Região Centro-Oeste $(6,4 \%)$ para o primeiro, e na Região Nordeste (6,5\%) para o segundo. Observou-se que os percentuais de agentes que referiram esses critérios foram bem superiores na zona rural (10,3\% e 9,4\%), em comparação com a zona urbana (2,5\% e $3,5 \%$ ) (figura 1).

Evidenciou-se que os critérios mais referidos pelos ACS desta pesquisa se enquadram nas recomendações da política de atenção domiciliar, haja vista que, em sua maioria, utilizaram: 'usuário com dificuldade de locomoção' (80,4\%); 'usuário idoso’ (74,9\%); e 'usuário com doença crônica' (71,3\%). O estudo de Jesus (2014) ${ }^{\mathbf{2 6}}$ encontrou resultados semelhantes ao revelar que os agentes priorizavam usuários com dificuldade de locomoção para realização das visitas ${ }^{26}$. Em outra investigação, verificou-se que, em cinco das 16 visitas realizadas, os agentes não possuíam clareza quanto ao motivo de sua realização ${ }^{38}$.

Ferreira ${ }^{16}$ evidenciou que as visitas domiciliares dos ACS ocorriam de forma 'engessada' e não seguiam critérios de risco e vulnerabilidade. Diante disso, o ACS concentra seu processo de trabalho sob recortes programáticos, em detrimento de critérios de necessidade dos usuários ${ }^{16}$. Outra pesquisa apontou que a gestão estadual e a gestão municipal consideram necessários critérios para as solicitações de visitas, definição do profissional que as realizará e mecanismos de avaliação. $\mathrm{Na}$ dinâmica de trabalho das equipes pesquisadas naquele estudo, as formas de organização foram diversas, e, portanto, umas favoreciam, outras dificultavam a realização das visitas domiciliares. Em suma, as visitas respondiam a uma demanda espontânea dos usuários ou a demandas internas dos serviços, e inexistiam critérios para sua realização ${ }^{41}$. 
Figura 1. Percentual de agentes comunitários que referiu ter critérios definidos para realização de visitas domiciliares e tipo de critério segundo Regiões, Brasil e localização da unidade de saúde, 2015

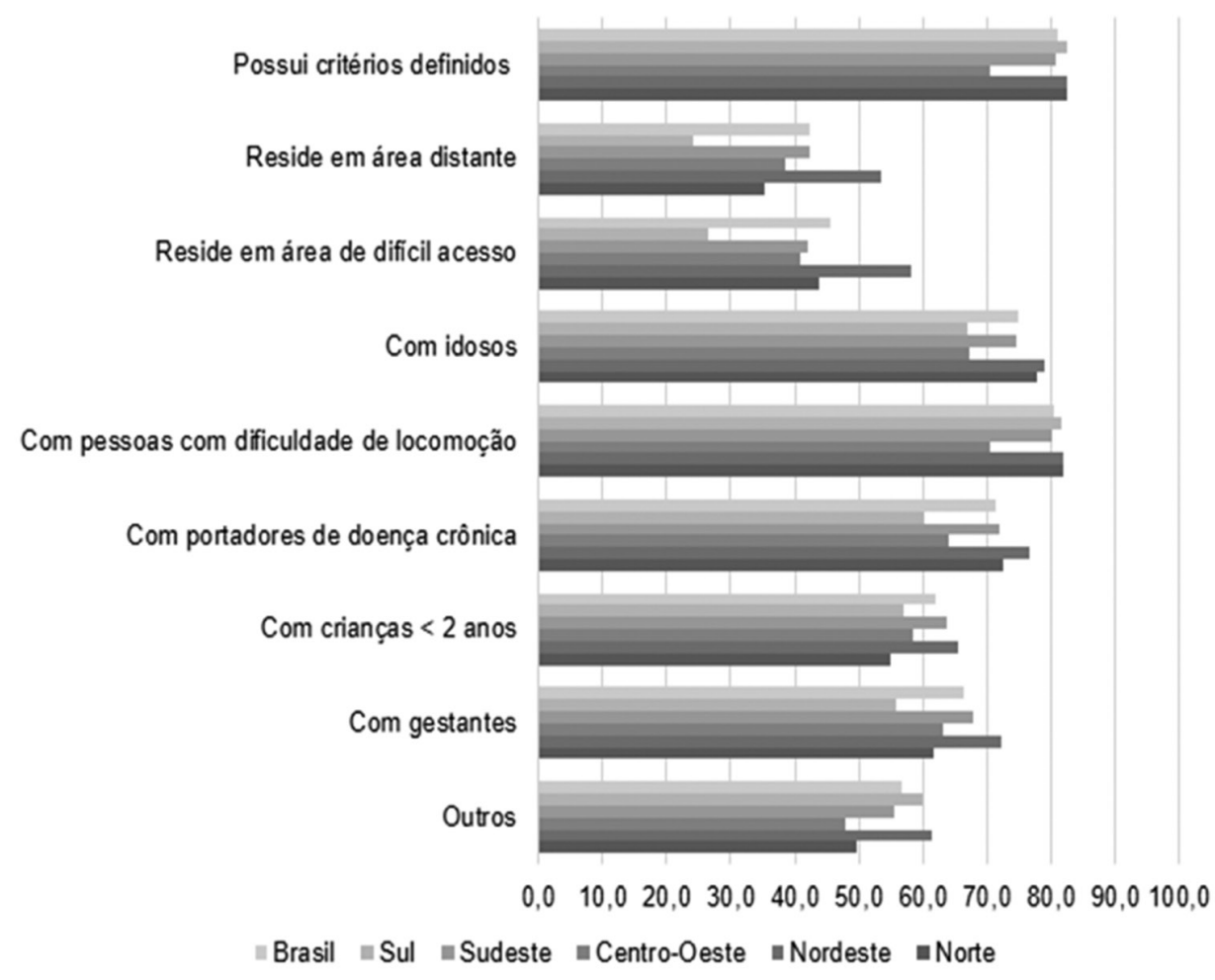

a. Brasil e Regiões

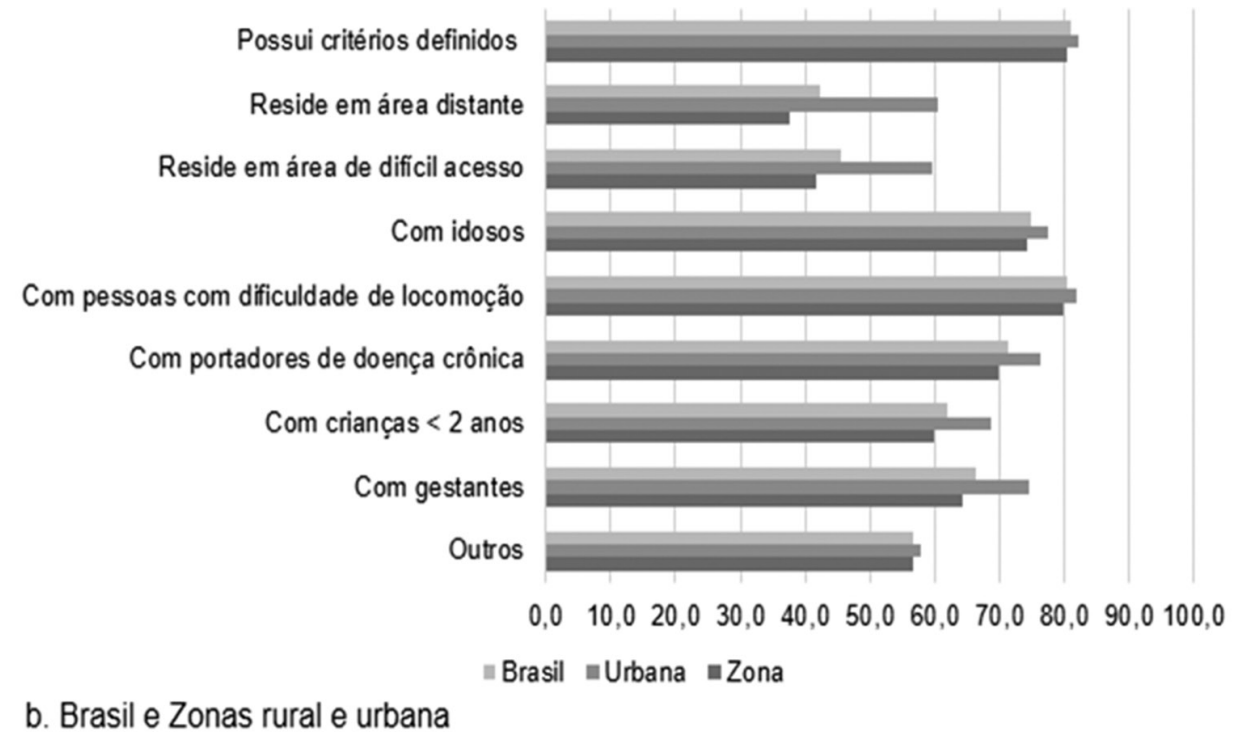


Com relação às atividades realizadas durante as visitas domiciliares, mais de $45 \%$ dos ACS referiram, espontaneamente, que orientavam sobre os serviços de saúde $(58,5 \%)$ e verificavam o cartão de vacinação (46,4\%); quase um terço levantava e acompanhava casos de doenças prevalentes (32,6\%); entre $20 \%$ a $25 \%$ entregavam medicamentos e orientavam quanto ao seu uso $(26,9 \%)$, pesavam as crianças $(21,9 \%)$ e cadastravam as famílias $(21,1 \%)$; e menos de $20 \%$ verificavam as condições ambientais (18,8\%), realizavam busca ativa de faltosos $(9,6 \%)$ e acompanhavam as famílias no $\operatorname{PBF}(8,8 \%)$ (figura 2).

A Região Sul apresentou os menores percentuais para a maioria das atividades (orienta sobre os serviços de saúde, verifica o cartão de vacinação, pesa as crianças, verifica as condições ambientais, realiza busca ativa de faltosos e acompanha as famílias no PBF), com exceção das atividades levanta e acompanha casos de doenças prevalentes $(38,8 \%)$, que exibiu o maior percentual, entrega medicamentos e orienta quanto ao uso $(23,3 \%)$ e cadastra as famílias $(25,6 \%)$. Os maiores percentuais foram observados na Região Norte para as atividades cadastra as famílias (29,1\%), verifica as condições ambientais (39,7\%), realiza busca ativa de faltosos (16,1\%) e acompanha as famílias no PBF (11,1\%); na Região Nordeste, para as atividades orienta sobre os serviços de saúde $(60,6 \%)$, verifica o cartão de vacinação $(51,0 \%)$ e pesa as crianças (41,3\%); e na Região Centro-Oeste, para a atividade entrega medicamentos e orienta quanto ao seu uso (36,0\%) (figura 2).

Na comparação urbano-rural, apenas em duas atividades foram observados maiores percentuais na zona rural $(63,4 \%$ orientavam sobre os serviços de saúde e $45,3 \%$ pesavam as crianças). Nas demais atividades, os percentuais foram superiores na zona urbana $(34,1 \%$ levantavam e acompanhavam casos de doenças prevalentes; $32,8 \%$ entregavam medicamentos e orientavam quanto ao seu uso; $22,9 \%$ cadastravam as famílias; $19,6 \%$ verificavam as condições ambientais; $10,1 \%$ realizavam busca ativa de faltosos e 9,2\% acompanhavam as famílias no PBF). Não houve diferença nos percentuais quanto à atividade verifica cartão de vacinação (figura 2). 
Figura 2. Percentual de agentes comunitários, segundo tipo de atividade que referiu realizar durante as visitas domiciliares, de acordo com a localização da unidade de saúde, Regiões e Brasil, 2015

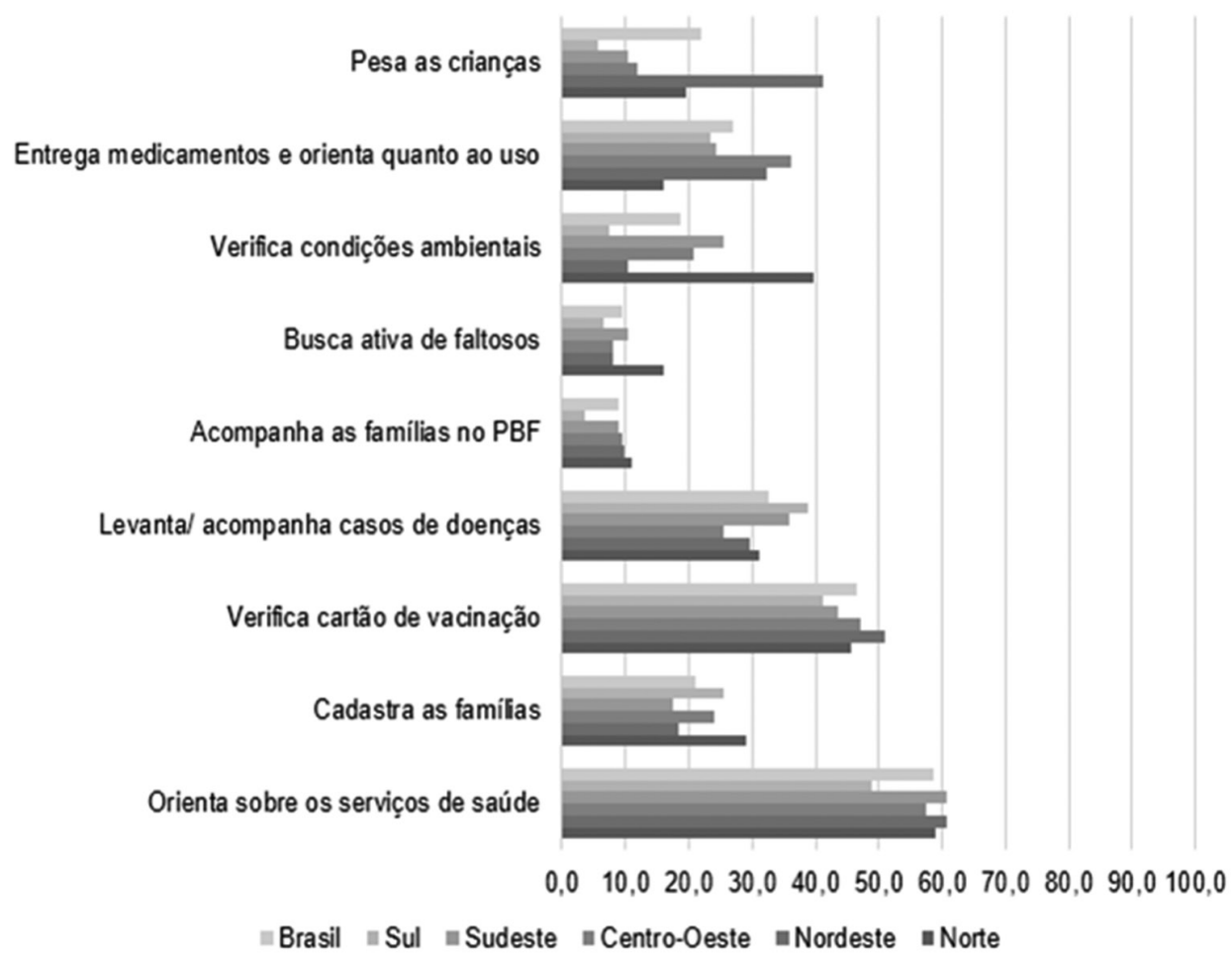

a. Brasil e Regiões

Pesa as crianças

Entrega medicamentos e orienta quanto ao uso

Verifica condições ambientais

Busca ativa de faltosos

Acompanha as famílias no PBF

Levanta/acompanha casos de doenças

Verifica cartão de vacinação

Cadastra as familias

Orienta sobre os serviços de saúde

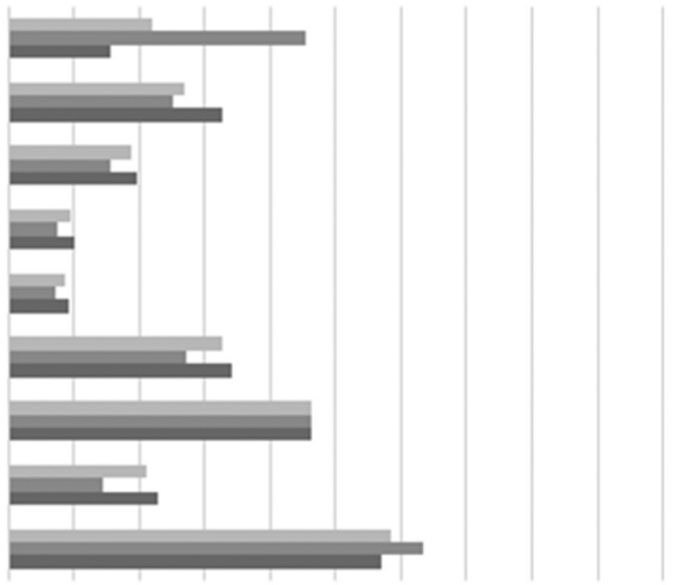

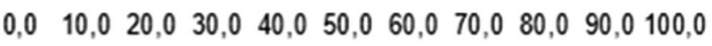

\section{\#rasil $=$ Rural $n$ Urbana}

b. Brasil e Zonas rural e urbana 
Alguns estudos encontraram resultados bastante similares ao deste inquérito, destacando que as atividades realizadas nas visitas domiciliares eram pautadas na orientação sobre o funcionamento das unidades de saúde, sobre o uso de medicação, ações de encaminhamento ao posto e verificação do cartão da criança ${ }^{24,35}$. Inquérito municipal realizado com 241 ACS evidenciou que a orientação da família quanto à utilização dos serviços de saúde e o encaminhamento e agendamento de consultas e exames foram mencionados pela grande maioria dos agentes, respectivamente, $99,6 \%$ e $78,8 \%{ }^{\mathbf{1 4}}$.

Outros achados indicaram que as práticas de saúde dos ACS durante a VD reafirmavam um modelo de atenção centrado na doença, cujas ações focavam orientações relativas à realização de exames, aos cuidados de pessoas enfermas e/ou crianças ${ }^{\mathbf{2 6}, 42}$. Outra pesquisa apontou uma expressiva relação de dependência do trabalho do ACS com a EqSF, situação que tem contribuído para um tímido protagonismo desse profissional nas intervenções sobre o território. Isso restringia as práticas durante as visitas domiciliares a uma espécie de supervisão dos cuidados propostos pelo médico, ainda assim, restrita a grupos específicos (hipertensos, diabéticos, crianças menores de dois anos, gestantes, tuberculosos, entre outros) ${ }^{43}$.

Nesse aspecto, Lara também identificou atividades centradas exclusivamente em grupos de risco (hipertensos e diabéticos), saúde da criança, gestantes, puérperas e idosos ${ }^{34}$. Ao contrário, outro estudo evidenciou práticas centradas na educação em saúde e orientação sobre uso dos serviços de saúde primários ${ }^{44}$. Alguns autores assinalam a educação em saúde como principal eixo de trabalho do ACS. Nessa perspectiva, o agente comunitário seria um ator social fundamental para promover mudanças e estimular a participação da comunidade ${ }^{45}$. Outros destacam a VD como instrumento facilitador do vínculo entre ACS, usuários e familiares ${ }^{46}$.

Estudo em município brasileiro evidenciou que 71,6\% dos ACS buscam, na visita, identificar a relação entre problemas de saúde e condições de vida dos usuários. Esse fato indicou visitas centradas nos problemas e nas condições de vida das famílias e da comunidade adscrita, diariamente ${ }^{47}$. No estudo de Gaíva e colaboradores $^{\mathbf{4 0}}$, também houve um alto índice de visitas centradas em grupos prioritários. Chama a atenção que os ACS referiram variar a abordagem durante as visitas, uma vez que é preciso considerar as características e necessidades ${ }^{40}$. Entretanto, houve unanimidade de maior dedicação às visitas a famílias com membros enfermos, reforçando, novamente, um modelo centrado na doença.

\section{Considerações finais}

Os resultados deste estudo, analisados à luz da PNAB 2011, indicam a realização periódica de visitas domiciliares, dirigidas ao monitoramento de grupos prioritários, a exemplo de gestantes de risco e crianças menores de um ano, com intervalo de até 15 dias. Ressalte-se, também, a mediana nacional de 150 famílias sob a responsabilidade de cada ACS.

Contudo, entre as atividades realizadas durante as visitas, chama atenção a pequena proporção da verificação das condições ambientais no domicílio, do acompanhamento das famílias vinculadas ao PBF e da busca ativa de faltosos, indicando um comprometimento da organização do processo de trabalho das equipes que incide sobre o escopo das visitas, atividade basilar dos ACS no Brasil.

Em setembro de 2017, uma nova revisão da PNAB, publicada sem o debate necessário de suas proposições, tem sido alvo de críticas em função de medidas restritivas que colocam em risco a organização da APS 
a partir da $\mathrm{ESF}^{\mathbf{4 8 , 4 9}}$. Entre outras medidas problematizadas, destaca-se a flexibilização da presença dos ACS na composição das EqSF, podendo acarretar a descontinuidade das ações focadas no território, comprometendo o acesso e a efetividade das ações de saúde. Certamente, os efeitos positivos da ESF sobre indicadores epidemiológicos demonstrados na literatura não seriam possíveis sem a presença do ACS no contato cotidiano com as famílias em seu território de atuação.

\section{Referências}

1. Aquino R, Medina MG, Nunes CA, et al. A Estratégia Saúde da Família e o reordenamento do sistema de serviços de saúde. In: Paim J, Almeida-Filho N, organizadores. Saúde Coletiva: Teoria e Prática. Rio de Janeiro: MedBooks; 2013.

2. Fausto MCR, Bousquat A, Lima JG, et al. Evaluation of Brazilian Primary Health Care From the Perspective of the Users: Accessible, Continuous, and Acceptable. J Ambulatory Care Manage [internet]. 2017 [acesso em 2018 jul 10]; 40(Supl.2):S60-S70. Disponível em: https://www.ncbi.nlm.nih. gov/pmc/articles/PMC5338884/pdf/jamcm-40-s60.pdf.

\section{Colaboradores}

Nunes CA e Aquino $\mathrm{R}$ foram responsáveis pela concepção, planejamento, análise e interpretação dos dados, elaboração do rascunho, revisão crítica do conteúdo e aprovação da versão final do manuscrito. Medina MG e Vilasbôas ALQ foram responsáveis pela concepção da pesquisa, produção, análise, interpretação dos dados e redação do artigo. Pinto Júnior EP e Luz L foram responsáveis pela análise e interpretação dos dados.

3. Macinko J. Evaluation of the impact of the Family Health Program on infant mortality in Brazil, 19902002. Journal Epidemiol Community Health [internet]. 2006 [acesso em 2018 jul 10]; 60(1):13-19. Disponível em: https://www.ncbi.nlm.nih.gov/pmc/ articles/PMC2465542/pdf/13.pdf.

4. Aquino R, Oliveira NF, Barreto ML. Impact of the Family Health Program on Infant Mortality in Brazilian Municipalities. Am J Public Health. 2009; 99(1):87-93.

5. Rasella D, Harhay MO, Pamponet ML, et al. Impact of primary health care on mortality from he- 
art and cerebrovascular diseases in Brazil: a nationwide analysis of longitudinal data. BMJ. 2014 jul; 349(35):4014-4024.

6. Dourado I, Oliveira VB, Aquino R, et al. Trends in Primary Health Care-sensitive Conditions in Brazil: The Role of the Family Health Program (Project ICSAP-Brazil). Medical Care. 2011 jun; 49(6):577-584.

7. Macinko J, Harris M. Brazil's Family Health Strategy: Delivering community based primary care in a universal health system. N Engl J Med [internet]. 2015 [acesso em 2018 jul 10]; 372(2):21772181. Disponível em: https://www.nejm.org/doi/ pdf/10.1056/NEJMp1501140.

8. Brasil. Ministério da Saúde. Portaria nº 1886/GM, de 18 de dezembro de 1997. Aprova as Normas e Diretrizes do Programa de Agentes Comunitários de Saúde e do Programa de Saúde da Família. Diário Oficial da União. 18 Dez 1997.

9. Brasil. Ministério da Saúde. Portaria no 2.488, de 21 de outubro de 2011. Aprova a Política Nacional de Atenção Básica, estabelecendo a revisão de diretrizes e normas para a organização da Atenção Básica, para a Estratégia Saúde da Família (ESF) e o Programa de Agentes Comunitários de Saúde (PACS). Diário Oficial da União. 21 Out 2011.

10. Brasil. Presidência da República. Lei no 10.507, de 10 de julho de 2002. Cria a profissão de Agente Comunitário de Saúde e dá outras providências. Diário Oficial da União. 10 Jul 2002.

11. Brasil. Presidência da República. Lei $\mathrm{n}^{\circ} 13.595$, de 5 de janeiro de 2018. Diário Oficial da União. 5 Jan 2018.

12. Alonso CMC, Béguin PD, Duarte FJCM. Trabalho dos agentes comunitários de saúde na Estratégia Saúde da Família: metassíntese. Rev Saúde Pública [internet]. $2018 \mathrm{fev}$ [acesso em 2018 jul 10]; 52(14):113. Disponível em: http://www.scielo.br/pdf/rsp/ v52/pt_0034-8910-rsp-S1518-87872018052000395. pdf.
13. Filgueiras AS, Silva ALA. Agente Comunitário de Saúde: um novo ator no cenário da saúde do Brasil. Physis [internet]. 2011 [acesso em 2018 jul 10]; 21(3):899-916. Disponível em: http://www.scielo. br/pdf/physis/v2ln3/08.pdf.

14. Costa SM, Araújo FF, Martins LV, et al. Agente Comunitário de Saúde: elemento nuclear das ações em saúde. Ciênc Saúde Colet [internet]. 2013 jul [acesso em 2018 jul 10]; 18(7):2147-2156. Disponível em: http://www.scielo.br/pdf/csc/v18n7/30.pdf.

15. Silva JA, Dalmaso ASW. O agente comunitário de saúde e suas atribuições: os desafios para os processos de formação de recursos humanos em saúde. Interface [internet]. $2002 \mathrm{fev}$ [acesso em 2018 jul 10]; 6(10):75-96. Disponível em: http://www.scielo.br/ pdf/icse/v6n10/07.pdf.

16. Ferreira VSC, Andrade CS, Franco TB, et al. Processo de trabalho do agente comunitário de saúde e a reestruturação produtiva. Cad Saúde Pública [internet]. 2009 [acesso em 2018 jul 10]; 25(4):898906. Disponível em: http://www.scielo.br/pdf/csp/ v25n4/21.pdf.

17. Galavote HS, Prado TN, Maciel ELN, et al. Desvendando os processos de trabalho do agente comunitário de saúde nos cenários revelados na Estratégia Saúde da Família no município de Vitória (ES, Brasil). Ciênc Saúde Colet [internet]. 2011 jan [acesso em 2018 jul 10]; 16(1):231-240. Disponível em: http://www.scielo.br/pdf/csc/v16nl/v16nla26.pdf.

18. Morosini MVGC. O agente comunitário de saúde: práticas educativas. Trab Educ Saúde [internet]. 2012 [acesso em 2018 jul 10]; 10(2):347-348. Disponível em: http://www.scielo.br/pdf/tes/v10n2/10. pdf.

19. Ursine BL, Trelha CS, Nunes EFPA. O Agente Comunitário de Saúde na Estratégia de Saúde da Família: uma investigação das condições de trabalho e da qualidade de vida. Rev Bras Saúde Ocup [internet]. $2010 \mathrm{dez}$ [acesso em 2018 jul 10]; 35(12):327339. Disponível em: http://www.scielo.br/pdf/rbso/ v35n122/a15v35n122.pdf. 
20. Simões AL, Freitas CM. Análise sobre condições de trabalho de Equipe de Saúde da Família, num contexto de vulnerabilidades, Manaus (AM). Saúde debate [internet]. 2016 [acesso em 2018 jul 10]; 40(109):47-58. Disponível em: http:// www.scielo.br/pdf/sdeb/v40n109/0103-1104sdeb-40-109-00047.pdf.

21. Gomes KO, Cotta RMM, Cherchiglia ML, et al. A práxis do agente comunitário de saúde no contexto do programa saúde da família: reflexões estratégicas. Saúde Soc [internet]. $2009 \mathrm{dez}$ [acesso em 2018 jul 10]; 18(4):744-755. Disponível em: http://www. scielo.br/pdf/sausoc/v18n4/17.pdf.

22. Nunes MO, Trad LB, Almeida BA, et al. O agente comunitário de saúde: construção da identidade desse personagem híbrido e polifônico. Cad Saúde Pública [internet]. $2002 \mathrm{dez}$ [acesso em 2018 jul 10]; 18(6):1639-1646. Disponível em: http://www.scielo. br/pdf/csp/v18n6/13260.pdf.

23. Lima AN, Silva L, Bousso RS. A visita domiciliária realizada pelo agente comunitário de saúde sob a ótica de adultos e idosos. Saúde Soc [internet]. 2010 dez [acesso em 2018 jul 10]; 19(4):889-897. Disponível em: http://www.scielo.br/pdf/sausoc/v19n4/15. pdf.

24. Kebian LVA, Acioli S. Visita domiciliar: espaço de práticas de cuidado do enfermeiro e do agente comunitário de saúde. Rev Enferm UERJ [internet]. 2011 [acesso em 2018 jul 10]; 19(3):403-409. Disponível em: http://www.facenf.uerj.br/v19n3/ v19n3all.pdf.

25. Amorim DU, Queiroz MVO, Brasil EGM, et al. Percepções e práticas de agentes comunitários de saúde sobre seu trabalho com adolescentes. Saúde debate [internet]. 2014 jun [acesso em 2018 jul 10]; 38(101):254-226. Disponível em: http://www.scielo. br/pdf/sdeb/v38n101/0103-1104-sdeb-38-101-0254. pdf.

26. Jesus AS, Santos FPA, Rodrigues VP, et al. Atuação do agente comunitário de saúde: conhecimento de usuários. Rev Enferm UERJ [internet]. 2014 mar-abr [acesso em 2018 jul 10]; 22(2):239-244. Disponível em: http://www.facenf.uerj.br/v22n2/ v22n2a15.pdf.

27. Hildebrand SM, Shimizu HE. Percepção do agente comunitário sobre o Programa Família Saudável. Rev Bras Enferm [internet]. 2008 jun [acesso em 2018 jul 10]; 61(3):319-324. Disponível em: http:// www.scielo.br/pdf/reben/v6ln3/a07v61n3.pdf.

28. Oliveira ELC, Krull LS, Figueredo SV. A visita domiciliar na estratégia saúde da família sob os aspectos do planejamento e da abordagem familiar [trabalho de conclusão de curso]. Rio de Janeiro: Fiocruz/ Ensp; 2009. 63 p.

29. Mandú ENT, Gaíva MAM, Silva MA, et al. Visita domiciliária sob o olhar de usuários do programa saúde da família. Texto Contexto Enferm [internet]. 2008 mar [acesso em 2018 jul 10]; 17(1):131-140. Disponível em: http://www.scielo.br/pdf/tce/v17nl/15. pdf.

30. Baralhas M, Pereira MAO. Concepções dos agentes comunitários de saúde sobre suas práticas assistenciais. Physis [internet]. 2011 [acesso em 2018 jul 10]; 21(1):31-46. Disponível: http://www.scielo.br/pdf/ physis/v2ln1/v2ln1a02.pdf.

31. Bousquat A, Giovanella L, Fausto MCR, et al. Tipologia da estrutura das unidades básicas de saúde brasileiras: os 5 R. Cad Saúde Pública [internet]. 2017 [acesso em 2018 jul 10]; 33(8):1-15. Disponível em: http://www.scielo.br/pdf/csp/v33n8/16784464-csp-33-08-e00037316.pdf.

32. Saliba NA, Garbin CAS, Silva FSJFB, et al. Agente comunitário de saúde: perfil e protagonismo na consolidação da atenção primária à saúde. Cad Saúde Colet [internet] 2011 [acesso em 2018 jul 10]; 19(3):318-326. Disponível em: http://www.cadernos.iesc.ufrj.br/cadernos/images/csc/2011_3/artigos/csc_v19n3_318-326.pdf.

33. Fracoli LA, Almeida EZ. Teoria e prática da promoção da saúde: as concepções dos agentes comunitários de saúde. Mundo Saúde [internet]. 2011 [aces- 
so em 2018 jul 10]; 35(2):137-144. Disponível em: http://bvsms.saude.gov.br/bvs/artigos/teoria_pratica_promocao_saude.pdf.

34. Oliveira LM, Menezes BMJ, Cristina RL. Aspectos culturais das práticas dos Agentes Comunitários de Saúde em áreas rurais. Rev Esc Enferm USP [internet]. 2012 [acesso em 2018 jul 10]; 46(3):673-680. Disponível em: http://www.scielo.br/pdf/reeusp/ v46n3/20.pdf.

35. Oliveira LS, Nachif MCA, Matheus MLF. O trabalho do agente comunitário de saúde na percepção da comunidade de Anastácio, Estado do Mato Grosso do Sul. Health Sciences. 2003 [acesso em 2018 jul 10]; 25(1)95-101. Disponível em: http://www.periodicos.uem.br/ojs/index.php/ActaSciHealthSci/article/view/2306/1478.

36. Sakata KN, Almeida MCP, Alvarenga AM, et al. Concepções da equipe de saúde da família sobre as visitas domiciliares. Rev Bras Enferm [internet]. 2007 dez [acesso em 2018 jul 10]; 60(6):659-664. Disponível em: http://www.scielo.br/pdf/reben/v60n6/07. pdf.

37. Kebian LVA, Acioli S. A visita domiciliar de enfermeiros e agentes comunitários de saúde da Estratégia Saúde da Família. Rev Eletr Enf [internet]. 2014 jan-mar [acesso em 2018 jul 10]; 16(1):161-169. Disponível em: https://revistas.ufg.br/fen/article/ view/20260/16455.

38. Alves MP, Santos SSC. Um olhar sobre o trabalho dos agentes comunitários de saúde: a visita domiciliar. Rev Baiana Enferm [internet]. 2007 jan-abr [acesso em 2018 jul 10]; 21(1):71-79. Disponível em: https://portalseer.ufba.br/index.php/enfermagem/ article/view/3914/2889.

39. Moreira TNF, Martins CL, Feuerwerker LCM, et al. The foundation of care: Family Health Program teams dealing with domestic violence situations. Saúde Soc [internet]. 2014 set [acesso em 2018 jul 10]; 23(3):814-827. Disponível em: http://www.scielo. br/pdf/sausoc/v23n3/0104-1290-sausoc-23-3-0814. pdf.
40. Gaíva MAM, Siqueira VCA. A prática da visita domiciliária pelos profissionais da estratégia saúde da família. Ciênc Cuid Saúde [internet]. 2011 [acesso em 2018 jul 10]; 10(4):697-704. Disponível em: http://periodicos.uem.br/ojs/index.php/CiencCuidSaude/article/viewFile/18313/pdf.

41. Mendonça MHM. Agente comunitário de saúde: o ser, o saber, o fazer. Cad Saúde Pública [internet]. 2004 out [acesso em 2018 jul 10]; 20(5):1433-1434. Disponível em: http://www.scielo.br/pdf/csp/ v20n5/41.pdf.

42. Pereira IC, Oliveira MAC. O trabalho do agente comunitário na promoção da saúde: revisão integrativa da literatura. Rev Bras Enferm [internet]. 2013 jun [acesso em 2018 jul 10]; 66(3):412-419. Disponível em: http://www.scielo.br/pdf/reben/v66n3/ al7v66n3.pdf

43. Silva NC, Andrade CS. Agente comunitário de saúde: questões ambientais e promoção da saúde em comunidades ribeirinhas. Trab Educ Saúde [internet]. 2013 [acesso em 2018 jul 10]; 11(1)113-128. Disponível em: http://www.scielo.br/pdf/tes/vllnl/ a07v1lnl.pdf.

44. Lanzoni GMM, Cechinel C, Meirelles BHS. Agente Comunitário de Saúde: estratégias e consequências da sua rede de relações e interações. Rev Rene [internet]. 2014 [acesso em 2018 jul 10]; 15(1):123-133. Disponível em: http://periodicos.ufc.br/rene/article/view/3096/pdf.

45. Fonseca AF, Morosini MVGC, Mendonça MHM. Atenção primária à saúde e o perfil social do trabalhador comunitário em perspectiva histórica. Trab Educ Saúde [internet]. 2013 [acesso em 2018 jul 10]; 11(3):525-552. Disponível em: http://www.scielo. br/pdf/tes/v1ln3/v1ln3a05.pdf.

46. Rodrigues AÁAO, Santos AM, Assis MMA. Agente comunitário de saúde: sujeito da prática em saúde bucal em Alagoinhas, Bahia. Ciênc Saúde Colet [internet]. 2010 maio [acesso em 2018 jul 10]; 15(3):907-915. Disponível em: http://www.scielo.br/ pdf/csc/v15n3/v15n3a34.pdf. 
47. Fracolli LA, Gomes MFP, Pinheiro DGM, et al. Avaliação das ações de promoção da saúde desenvolvidas pelos agentes comunitários de saúde. Mundo Saúde [internet]. 2013 [acesso em 2018 jul 10]; 37(4):411-418. Disponível em: http://bvsms.saude. gov.br/bvs/artigos/mundo_saude/avaliacao_acoes_ promocao_saude_desenvolvidas.pdf.

48. Morosini MVGC, Fonseca AF. Reviewing the Brazilian National Primary Healthcare Policy at such a time? Cad Saúde Pública. 2017; 33(1):1-3.
49. Morosini MVGC, Fonseca AF, Lima LD. Política Nacional de Atenção Básica 2017: retrocessos e riscos para o Sistema Único de Saúde. Saúde debate [internet]. 2018 jan [acesso em 2018 jul 10]; 42(116):1124. Disponível em: http://www.scielo.br/pdf/sdeb/ v42n116/0103-1104-sdeb-42-116-0011.pdf.

Recebido em 13/07/2018

Aprovado em 19/09/2018

Conflito de interesses: inexistente

Suporte financeiro: Ministério da Saúde 